\title{
Comparison of the fragmentation behavior of DNA and LNA single strands and duplexes
}

Stefanie Ickert 1,2, Timm Schwaar 2, Andreas Springer 3, Márkó Grabarics ${ }^{4}$, Jens Riedel 2, Sebastian Beck 1, Kevin Pagel 3,4, Michael W. Linscheid 1,*

${ }^{1}$ Humboldt-Universitaet zu Berlin, Department of Chemistry, Berlin, Germany

${ }^{2}$ Federal Institute for Materials Research and Testing, Berlin, Germany

${ }^{3}$ Freie Universitaet Berlin, Department of Chemistry and Biochemistry, Berlin, Germany

${ }^{4}$ Fritz Haber Institute of the Max Planck Society, Department of Molecular Physics, Berlin, Germany

*corresponding author: email: m.linscheid@chemie.hu-berlin.de, phone: +49(0)30 209366471 , Humboldt-Universitaet zu Berlin, Department of Chemistry, Unter den Linden 6, 10099 Berlin, Germany

Keywords: oligonucleotide fragmentation, locked nucleic acids, collision induced dissociation (CID), double strands, ion mobility spectrometry

\begin{abstract}
DNA and locked nucleic acid (LNA) were characterized as single strands, as well as double stranded DNA-DNA duplexes and DNA-LNA hybrids using tandem mass spectrometry with collision-induced dissociation. Additionally, ion mobility spectrometry was carried out on selected species. Oligonucleotide duplexes of different sequences - bearing mismatch positions and abasic sites of complementary DNA 15-mers - were investigated to unravel general trends in their stability in the gas phase. Single stranded LNA oligonucleotides were also investigated with respect to their gas phase behavior and fragmentation upon collision-induced dissociation. In contrast to the collision-induced dissociation of DNA, almost no base loss was observed for LNAs. Here, backbone cleavages were the dominant dissociation pathways. This finding was further underlined by the need for higher activation energies. Base losses from the LNA strand
\end{abstract}


were also absent in fragmentation experiments of the investigated DNA-LNA hybrid duplexes. While DNA-DNA duplexes dissociated easily into single stranded fragments, the high stability of DNA-LNA hybrids resulted in predominant fragmentation of the DNA part rather than the LNA, while base losses were only observed from the DNA single strand of the hybrid.

\section{Introduction}

The fragmentation behavior of DNA oligonucleotides (ONs) in mass spectrometry (MS) has been widely investigated before, while a specific nomenclature for fragments has been introduced at an early stage (Figure 1). ${ }^{1}$ Influences of base identity, sequence and charge state have been described for collision-induced dissociation (CID) under soft ionization conditions. ${ }^{1-6}$

Generally, the loss of a base (either neutral or charged) is the predominant fragmentation pathway and there is a great tendency for adenine loss, whereas guanine loss is less favored. Besides this preferred dissociation channel, $\mathrm{w}$ and $\mathrm{a}_{\mathrm{x}}-\mathrm{B}$ fragments are generally of high abundance in DNA CID fragment spectra. In RNA SSs mainly c- and y-type ions are formed besides base losses upon CID activation. ${ }^{1,7,8}$

Other ion activation methods such as infrared multiphoton dissociation (IRMPD), blackbody infrared radiative dissociation (BIRD) or higher energy collisional dissociation (HCD), were also employed to study the fragmentation of DNA ONs. Similar fragmentation behavior were observed for DNAs employing these activation conditions. ${ }^{9-13}$ In kinetically driven electron-based fragmentation activations like electron-capture dissociation (ECD) and electron-transfer dissociation (ETD) with positively charged precursor ions, complementary ions are formed. In both, sequence specific backbone cleavages are predominant, leading to a-, d-, w- and z-series ions, while radical species ( $\mathrm{a}-\mathrm{z}$ - - type) and $\mathrm{a}-\mathrm{B} / \mathrm{z}-\mathrm{B} / \mathrm{c}-\mathrm{B} / \mathrm{x}-\mathrm{B}$ fragment ions are formed to a minor extend. ${ }^{14-17}$ While the majority of these studies investigated SS DNA and RNA nucleic acids, only limited information is available for double stranded (DS) nucleic acids. ${ }^{18,19}$ Gabelica et al. compared the CID-derived spectra of four different charge states of a 16-mer DNA duplex. 18 In the charge states 8-, 7- and 6- preferably the separation into SSs was detected. Only at the 
lowest investigated charge state of 5- were base losses and single strand fragments observed. Apart from these two studies, no DS DNA fragmentation behavior has been investigated.

Ion mobility-mass spectrometry (IM-MS) was also used previously to uncover DS properties. ${ }^{20,21}$ Porrini et al. recently discussed the transition of DS nucleic acids from solution to the gas phase. They found a high dependency of collisional cross-sections (CCSs) on the charge state, while in general more compact structures were found in the gas phase in comparison to the solution structures. ${ }^{22}$ This can be related to the A-type helix the DS forms in the gas phase, in difference to the water-rich B-type helix structure in solution.22,23 This gas phase structure was also found for shorter DNA DS sequences, ${ }^{23}$ while for ONs not long enough to form such A-type helices (4mers, 6-mers), also globular structures with less defined folding were reported.24

RNA and DNA analogs came into focus during the last few decades for ON-based therapeutics, as their non-toxic nature and higher resistance toward rapid enzymatic digestion by endo- and exonucleases made them ideal candidates for therapeutic purposes. ${ }^{25-27}$ One of these analogues is locked nucleic acid (LNA), harboring an additional methylene bridge between the 2 ' oxygen and $4^{\prime}$ carbon atom of the ribose unit (Figure 2).28-30 This chemically stable methylene bridge locking the ribose in the $3^{\prime}$-endo conformation - increases the melting temperature of LNAcontaining duplexes compared to their RNA/DNA counterparts, while having similar physicochemical properties. The structure of LNAs was studied extensively using nuclear magnetic resonance (NMR) spectroscopy and X-ray crystallography, but little attention has been paid to the gas phase characterization of SS LNAs or DNA-LNA hybrids by MS. Only one reference is available that investigates the gas phase behavior of LNAs using MS. ${ }^{31}$ Even in this reference, mixed DNA and LNA monomers were used for synthesis of oligonucleotides.

In the present study, we investigated the CID fragmentation behavior of SS DNA and LNA ONs and compared the results to corresponding DS ONs. Additionally, selected ion species were subjected to IM-MS analyses to determine their CCSs, reflecting the overall shape and size of the nucleic acid ions.

\section{Materials \& Methods}


All DNA ONs were purchased from BioTeZ Berlin-Buch GmbH. LNA ONs, exclusively containing locked nucleotides, were purchased from Eurogentec Deutschland GmbH. Other chemicals were supplied by J.T. Baker and Carl Roth.

The SS ONs were dissolved in a solution of $30 \%$ methanol and $1 \%$ trimethylamine in water in a concentration of $10 \mu \mathrm{M}$. DS ONs were subjected to MS analyses dissolved in a solution of $0.2 \%$ triethylamine, $20 \%$ isopropanol and $50 \mathrm{mM}$ ammonium acetate in water with a final concentration of $10 \mu \mathrm{M}$. DNA-DNA and DNA-LNA DSs were prepared by mixing stoichiometrically equal amounts of corresponding SS ONs $(10 \mu \mathrm{M}$ per strand final concentration), heated to $95^{\circ} \mathrm{C}$ for $10 \mathrm{~min}$ and allowed to cool to room temperature over a period of $30 \mathrm{~min}$.

Negative ion ESI-MS spectra were obtained using an LTQ Orbitrap XL (Thermo Fisher Scientific). The instrument parameters were tuned automatically according to the maximum intensity of each signal investigated. Fixed instrumental parameters were as follows: $100^{\circ} \mathrm{C} /$ $400^{\circ} \mathrm{C}$ (SS/DS) transfer capillary temperature and 30 arbitrary units of nitrogen sheath gas flow. Samples were directly infused at a flow rate of $10 \mu \mathrm{L} \cdot \mathrm{min}^{-1}$ and a concentration of $10 \mu \mathrm{M}$. The precursor ions were isolated for CID experiments with an isolation width of $\Delta m / z=3$ and fragmented with an activation time of $30 \mathrm{~ms}$. Furthermore, a standard Synapt G2-S HDMS (Waters Corporation) was used to generate CID spectra. ESI spray voltage was set to $1.99 \mathrm{kV}$, source temperature and desolvation temperature were set to $100^{\circ} \mathrm{C}$ and $150^{\circ} \mathrm{C}$, respectively. Cone voltage and source offset were maintained at $40 \mathrm{~V}$. LM and HM resolution were 17 for CID experiments, the transfer collision cell remained at $4 \mathrm{~V}$, while the trap collision cell for activation was optimized for maximum abundance of the fragment ions. Both instruments were used to assess all sequences and theoretical fragments were calculated automatically. Therefore, we developed a JAVA run script termed DNA-/LNA-fragmentation_GUI in-house, to calculate the fragment masses of DNA and LNA SSs and DSs. The software is available by contacting the developer Timm Schwaar (timm.schwaar@gmx.de).

IM-MS analyses were performed using a modified Synapt G2-S HDMS, equipped with a linear drift tube following a design reported previously. 32 A nano-electrospray source (nESI) was used to typically ionize $5 \mu \mathrm{L}$ of sample from platinum-palladium-coated borosilicate emitters, prepared in-house. CCSs were determined using helium as drift gas. Parameters were the 
following: capillary voltage $1.0 \mathrm{kV}$, sampling cone $50 \mathrm{~V}$, source offset $25 \mathrm{~V}$, source temperature $30^{\circ} \mathrm{C}$, trap gas flow $2 \mathrm{~mL} \cdot \mathrm{min}^{-1}$. Ion mobility parameters were: IMS gas 2.2 torr of helium, trap DC entrance $2.0 \mathrm{~V}$, trap DC bias $5.0 \mathrm{~V}$, trap DC $-4.0 \mathrm{~V}$, trap DC exit 1.0 V, IMS DC entrance $-20.0 \mathrm{~V}$, helium cell DC 60.0 V, helium exit -40.0 V, IMS bias 50.0-140.0 V, transfer DC entrance 5.0 V, transfer DC exit $15.0 \mathrm{~V}$, trap wave velocity $250 \mathrm{~m} \cdot \mathrm{s}^{-1}$, trap wave height $15.0 \mathrm{~V}$, transfer wave velocity $300 \mathrm{~m} \cdot \mathrm{s}^{-1}$, transfer wave height $10.0 \mathrm{~V}$. IM-MS experiments were performed in negative ion mode and CCSs were determined using the stepped field method, where arrival time distributions were fitted using Gaussian functions and the centroid drift times were plotted versus the reciprocal drift voltages, as described elsewhere. ${ }^{32-34}$

\section{Results \& Discussion}

CID experiments of LNA single strands

As CID experiments of DNA SSs have widely been conducted, 1,2,4-6 only LNA SS ONs were examined under CID conditions. Huang et al. investigated short (one 5-mer and one 8-mer) LNAs, as well as one gapmer and one mixmer, the latter containing DNA and LNA monomers. They detected complete fragment series, ranging from a- to d-type ions and also w- to z-type ions. Furthermore, abundant base losses were observed. ${ }^{31}$ In contrast to the previous study, we employed larger oligomers solely containing LNA monomers.

At first, we examined four different 15-mers (Supplementary Information) all exclusively containing locked nucleotides and determined the characteristic fragmentation point (FP) of LNA ONs, which was previously described for DNA. ${ }^{35}$ An almost linear behavior was observed for the relation between FP and charge state, accompanied with an increase in relative collision energy (CE) by $1.3 \%$ to $3.1 \%$ in comparison to the DNA ONs of the same sequence (Figure 3a). Charge state 9- was an exception in this general behavior, as it required $1.0 \%$ less energy compared to the DNA ON. This phenomenon was caused by the previously described unique inflection point in DNA, ${ }^{35}$ which stems from the additional energy required for structural reorganization of DNA. This inflection point was not observed in any of the ONs of the rigid LNA structure. The results 
from the analyses of the other ONs (A1, C1, H1) are given in the Supplementary Information (Figures S1-S3).

With regards to the general fragmentation behavior, LNA fragments were very different from their DNA counterparts. The corresponding CID spectra exhibit a much higher number of fragments as in case of DNA. This appeared in all investigated ONs. For example, in the LNA ON F1, two to 4.5 times as many signals with a relative intensity of more than $5 \%$ were detected than for the corresponding DNA ON (Table 1). All other investigated LNA ONs exhibited a similar behavior. This high number of fragments most likely originated from the hindrance of base loss due to the additional methylene bridging in LNA. The lack of structural flexibility appears to hamper the previously described rearrangement, necessary for the elimination of a base. In DNA, simple base loss is the most favored fragmentation channel, resulting in few but abundant fragments. All other fragmentation pathways have higher activation energies. As the base loss was apparently hindered in LNA, the precursors dissociated via other fragmentation channels once their respective threshold energy was reached. As a result, multiple backbone cleavages occurred, resulting in a much larger number of fragments. In order to understand the differences between LNA and DNA fragmentation in more detail, additional 4-mer LNA ONs (Supplementary Information) were investigated. This step was necessary in order to understand the differences of LNA to DNA fragmentation in rather simple ON systems, before investigating more complex systems like hybrid duplexes. The differences in the resulting fragment patterns of both, DNA and LNA ONs, were also clearly observed here (Figure 4). In all investigated DNA 4mer ONs the dissociation preferences were similar and as previously reported. ${ }^{1}$ In particular, the simple base loss, $w$ and $a_{x}-B$ fragments were always of high abundance. The LNA 4-mer ONs again showed a different picture. In contrast to the previously described abundant base loss for mixmer and gapmer LNA ONs, ${ }^{31}$ the simple base loss occurred only with very low abundance, if any base loss was apparent at all. At the same time, hardly any signal of the singly negatively charged nucleobases was observed, which were of significant intensity in the DNA spectra. Instead, almost exclusively simple backbone cleavages without pronounced subsequent base losses were detected in LNA ONs. The LNA ONs showed no preference for a specific backbone cleavage position, as all fragments of a- to d- and w- to z-type were detected in high abundances (Figure 4). 


\section{CID experiments of DNA-DNA duplexes}

The effect of the charge state on the fragmentation behavior of DNA-DNA DSs has been studied in the past and the observed separation of the DS into SSs, was explained with the employed activation method and an increased Coulomb repulsion. ${ }^{18-20}$ As very little additional information is available on the fragmentation behavior of DNA-DNA DSs, we investigated the fragmentation behavior of these duplexes to compare them to the later described DNA-LNA duplexes. Three charge states of an 8-mer DNA DS ONs (Supplementary Information) were investigated. In contrast to the previous studies, ${ }^{18,19}$ where only a minor effect of the charge state was observed, we observed larger differences in our experiments. Previously, mostly DS separation, leading to SS fragments and simple base losses were observed. ${ }^{18,19}$ In our experiments, each of the three charge states showed a significantly different fragmentation behavior (Figure 5). In the fragment spectrum of $[\mathrm{M}-5 \mathrm{H}]^{5-}$ of the DS 8-mer1 and 8-mer2 (Supplementary Information) that was the highest observable charge state, a clear separation of the SSs was observed, which has been reported before for DNA-DNA duplexes. ${ }^{36}$ Both SSs were detected in the 2- and 3- charge states as dominant signals (Figure 5a), while the distribution of the charges on both strands appeared to be equally balanced. The CID spectrum of the 4- charge state mainly showed the separation of the two SSs upon CID as well (Figure 5b). Again, the charge distribution appeared balanced between the two strands, as other charge combinations (e.g. 3- and 1-) could not be detected. Although breakdown fragments of the SSs were observed in the 4- case, they were only of low abundance.

The CID spectrum of the DNA-DNA DS in the 3- charge state was fundamentally different. Although the separation into intact SSs with charges of 2- and 1- was apparent, a multitude of additional fragments was detected (Figure 5c and 5d). These fragments represented ions in which only smaller moieties such as bases were lost from the precursor ions, while the remaining DS was still intact (Table S1, Supplementary Information). These signals were not detected in the higher charge states of 5- and 4-.

While the dependence on the GC content has been described before, ${ }^{37,38}$ we further investigated the influence of base pairing and sequences towards the observed, previously unreported 
differences in the fragment spectra of different charge states. Eight different 15-mer DNA-DNA DSs with GC contents ranging between $0 \%$ and 100\% (DSs A-H, Supplementary Information) were employed. At first, general fragment formation with respect to type of fragments was examined. Similar results were obtained for all duplexes, while the DNA-DNA duplex F (F1+F2) shall be discussed here in more detail (Figures $6 \mathrm{a}$ and $6 \mathrm{~b}$ ). In the CID-derived fragment spectrum of the five-fold negatively charged precursor ions, in between $\mathrm{m} / \mathrm{z} 1600$ and $\mathrm{m} / \mathrm{z} 2000$, almost exclusively fragments of the DS were found and very few low abundant fragments of both SSs were detected. The simple base loss always represented the most abundant signal. In contrast to SS fragmentation, no thymine loss could be found in any DS fragment spectrum. Even when employing a 15-mer DS harboring a terminal thymine at one of the 3 ' ends, no thymine loss could be detected. The trend for base losses in all DS DNA ONs was in the order G $>\mathrm{A}>>\mathrm{C}>>>\mathrm{T}$. In general, with a GC content between 0 and $40 \%$, the adenine loss was predominant. With $60-100 \%$ GC contend, guanine loss became the dominant base loss, while a minor loss of cytosine could also be observed. In all investigated DSs loss of two bases could be unambiguously identified. Again, A and G losses were dominant in these cases. Moreover, even fragments generated by three base losses were observed. Most intense in this case was a fragment type were a terminal nucleoside (base and sugar) was lost. Furthermore, a cleavage in the second phosphate moiety accompanied with loss of a complete nucleotide and the adjacent nucleoside was also observed without abolishing non-covalent binding of the duplex. Cleavages in the progressing strand beyond the second phosphate unit could not be detected. The most frequent fragment class, although not very abundant, were fragments of the $[\mathrm{DS}-5 \mathrm{H}-\mathrm{N}-1 \mathrm{~B}]^{5-}$-type (one complete SS together with a fragment of the other strand that contains 14 nucleotides, $\mathrm{N}$ represents a nucleotide and $\mathrm{B}$ stands for nucleobase), where one nucleotide and a base was lost from the DS. Unfortunately, the clear assignment was hampered here by numerous overlaps of theoretical fragments in particular of the same class. In addition, fragments of the class [DS-5H$\mathrm{N}-2 \mathrm{~B}]^{5-}$ could be detected in the fragment spectra, albeit these were of lower abundance than those of the $[\mathrm{DS}-5 \mathrm{H}-\mathrm{N}-1 \mathrm{~B}]^{5-}$ class. In the range from $\mathrm{m} / \mathrm{z} 500$ to about $\mathrm{m} / \mathrm{z} 1600$, numerous fragments of the SSs were detected, which were assigned to a separation of the SSs with subsequent breakdowns (represented by numbers). The distribution of detected fragments among the two SSs was largely the same and was also observed in the examined charge states 1- and 2-. 
Mainly, w-type fragments and strand breaks accompanied by base losses were observed here. All experiments were repeated on the Synapt G2-S HDMS and both instruments generated very similar spectra except for the number of observed charge states.

\section{Artificial defects in DNA-DNA duplexes}

DSs with artificial defects were also investigated. Therefore, four DNA DS ONs with abasic sites (F1+F3-F6, Supplementary Information) were subjected to CID fragmentation in the observed charge states. We found that there was no relevance of localization of the abasic site towards the resulting fragment classes. The fragmentation pattern did not change significantly depending on whether the defect was at the end or in the middle of the DSs. However, increased intensities of base loss from the position opposing the defect were detected. This base is unable to form hydrogen bonds and was cleaved off readily upon CID activation in all charge states. In addition, almost no [DS-5H-2N-1B] $]^{5-}$ and $[\mathrm{DS}-5 \mathrm{H}-\mathrm{N}-2 \mathrm{~B}]^{5-}$-type fragments from the [DS-5H $]^{5-}$ precursor ion, as in the intact DNA-DNA DSs, were detected. Only for DS F1+F5 it was possible to detect a $[\mathrm{DS}-5 \mathrm{H}-\mathrm{N}-2 \mathrm{~B}]^{5-}-\mathrm{typ}$ fragment $\left(\left[\mathrm{F} 5+\mathrm{F}_{1} \mathrm{w}_{14-2} \mathrm{G}\right]^{5-}\right)$ with low intensity. In this case the fragmentation was potentially favored by a general destabilization of the duplex by the abasic site, as here also a higher preference for SS separation than in the other DSs was observed. This consideration was also supported by the fact that hardly any DS signals were detected in the survey spectra in general, as soon as two defects were located within the DSs. While, as mentioned above, DS fragments with three base losses were detected without destabilizing the DS within the MS, hardly any DSs could be detected in survey spectra, when two or more abasic sites were present. Surprisingly, this behavior did not occur when mismatches instead of abasic sites were employed. From the investigated four mismatch DSs (F1+F7-F10, Supplementary Information), containing one or two mismatches in various locations, increases in base losses were observed. In the case of AG mismatch $(\mathrm{F} 1+\mathrm{F} 7)$ within the strand, an increase in adenine base loss and a significant increase in double guanine loss could be observed. At a terminal CT mismatch $(\mathrm{F} 1+\mathrm{F} 9)$, no changes in the spectrum in comparison to the intact DS $(\mathrm{F} 1+\mathrm{F} 2)$ were found. Surprisingly, no thymine loss fragments could be generated despite a terminal destabilized thymine base (F1+F9). Only with a two-fold mismatch (F1+F10) it was possible to detect the 
neutral thymine loss fragment with low abundance. Overall, significantly fewer fragments could be generated for all mismatched DSs in relation to the intact DSs.

\section{CID experiments of DNA-LNA duplexes}

As a next step, we investigated DS DNA-LNA hybrids. For this purpose, the complementary 15mers (A1, C2, F1, H1, Supplementary Information) with the identical sequences as in the DNADNA cases were hybridized with the corresponding DNA SS to DNA-LNA hybrid DSs. In comparison to DNA-DNA DSs, both strands dissociated at higher CEs in the hybrids. As all trends in DNA-LNA DSs were similar, the DNA-LNA hybrid strand F (LNA-F1+DNA-F2) shall be discussed exemplarily here (Figures $6 \mathrm{c}$ and $6 \mathrm{~d}$ ). In general, fewer fragments were generated from the DNA-LNA hybrid than in the DNA-DNA case and the LNA strands were rarely fragmented. The distribution of the different fragment classes was on the other side well comparable. In the higher $\mathrm{m} / \mathrm{z}$ range, only fragments with (partially) intact DSs were observed, while between $\mathrm{m} / \mathrm{z} 500$ to about $\mathrm{m} / \mathrm{z} 1650$ only fragments of the SSs were detected. As mentioned above, in the case of the DNA-DNA DSs, fragmentation of both SSs was in general evenly distributed between the two DNA SSs (Figure 6 a). In contrast here, the DNA-LNA hybrids showed mostly only SS fragments that were clearly assigned to the DNA strand or were among the few that could theoretically be derived from both (Figure 6c). Overall, the relative intensities of the hybrids' SS fragments were higher in comparison to the DNA-DNA DSs. In the case of base loss, it was not possible to distinguish between guanine from DNA or LNA strands. Cytosine on the other hand with its additional methyl group in LNA (being 5-Methylcytosine in LNA) would have been differentiable between the DNA and LNA strands. However, no corresponding loss of cytosine has been detected.

\section{Energy dependence of fragment formation in double strands}

During fragmentation experiments of the DNA-DNA and DNA-LNA duplexes, we noticed that the preference for fragment formation was also energy dependent and different to the corresponding SS ONs (Figure 7). DS fragments were initially formed at low CEs, which resulted from cleavages at the ends of the DSs. Furthermore, the preference for this base loss was also 
energy dependent and in contrast to the corresponding SSs. Guanine and also adenine losses were readily observed, while cytosine losses were only marginal. ${ }^{10}$ The simple loss of a guanine base could already be observed with an isolation of the precursor ion, applying no CE at all. Other DS fragments (e.g. $[\mathrm{M}-5 \mathrm{H}-2 \mathrm{G}]^{5-}$ ) were also dominant at low CEs, while fragments that involved a backbone cleavage, for example the DS fragment $[\mathrm{M}-5 \mathrm{H}-\mathrm{N}]^{5-}$, were much lower in intensity at low CEs. SS fragments (e.g. $\left[\mathrm{c}_{4}-\mathrm{C}\right]^{-}$or $\left[\mathrm{w}_{6} / \mathrm{d}_{6}\right]^{2-}$ ) detected at all CEs, only accounted for a small portion of the total fragment intensities. The fragment $[\mathrm{M}-5 \mathrm{H}-\mathrm{G}]^{5-}$ could be detected as the most abundant signal up to a $\mathrm{CE}$ of $30 \%$. With a further increase in $\mathrm{CE}$, the intensity of $[\mathrm{M}-5 \mathrm{H}-\mathrm{N}]^{5-}$ increased to $100 \%$ intensity at $60-80 \% \mathrm{CE}$, while this fragmentation was not observed above $\sim 160 \%$ CE. High intensities for fragments with double base losses or strand breakdowns could be observed above a CE of $40 \%$.

\section{Ion Mobility-Mass Spectrometry}

To study the influence of the LNA monomers on the structure in the gas phase of LNA ONs, CCSs of SS DNA and LNA ONs were determined as a function of the charge state using IM-MS. Four different SS LNA 15-mers (A1, C1, F1, H1, Supplementary Information), along with their corresponding DNA equivalents possessing the identical sequence, were subjected to IM-MS analyses. This experimental framework allowed us to study the influence of the backbone, as well as the primary structure on the charged-induced structural changes of SS ONs in comparison to the previous described results. 35

Figure 3a illustrates the transition from more compact structures (represented by smaller CCS values) to extended conformations with increased CCSs, exemplarily for SS F1. The results from the analyses of the other investigated ONs (A1, C1, H1) were similar (Figures S1-S3, Supplementary Information). The plots show the effect of the backbone on the charge-induced structural changes of SS ONs. A general trend of sigmoidal correlation in the CCS-charge state correlations was obtained for all investigated LNA ONs, similar to the DNA counterparts. SS LNA ONs possessed larger CCSs at the lowest and highest charge states observed, while DNA SSs exhibited comparably higher CCS values for intermediate charge states. However, it should be noted that the differences between DNA and LNA were rather small at a given charge state and the changes in between charge states outweighed the LNA-DNA differences by far. When 
comparing nucleic acid SSs of the same type (DNA with DNA or LNA with LNA) with different base sequences, the influence of the sequence on the CCSs was also more pronounced. A higher $\mathrm{GC}$ content in the base sequence lead to lower CCS values, corresponding to more compact structures, which has been described before. ${ }^{39,40}$ This phenomenon was in accordance with the larger number of possible hydrogen bonds and, thus, stronger interactions between $\mathrm{G}$ and $\mathrm{C}$ upon forming base interactions, in difference to the weaker interactions between $\mathrm{A}$ and $\mathrm{T}$. Nevertheless, the differences in CCSs in between DNA and LNA were lower than expected taking the structural rigidity into account.

\section{Conclusion}

In this study we used CID and IM-MS to investigate the gas phase behavior of LNA oligomers with various sequences in correlation to their DNA analogues; mismatches and abasic sites were employed to obtain insights into the influence of the structural features of DNA and LNA ONs with respect to rigidity and base pairing in duplex stability. The LNA SSs possessed no inflection point in the CE-charge state correlation trends (Figure 3a). This led us to determine the CCSs of SS LNAs, as we presumed a lower flexibility of LNA ONs. CCSs were higher in LNA than for the corresponding DNA structures. The known higher rigidity of the backbone in LNA targets towards hampering any larger (re)folding in the LNA case. Nevertheless, the small differences determined do not explain the vastly different fragmentation behavior.

With SS DNA ONs the well-described behavior for base loss and subsequent backbone fragmentation was confirmed, as well as the previously shown influence of the number of negative charges. Unlike DNA ONs, LNA ONs showed a completely different dissociation behavior. Almost no base losses were observed and no preference for the frequently generated abundant backbone cleavages was found.

In DNA-DNA duplexes we found a clear preference for loss of a single base, additionally, a second and even a third base could be cleaved off as well. This was often followed by specific backbone cleavages with and without additional base losses. The separation of the duplexes into SSs proved to be a function of applied CE and charge states as expected. DNA-LNA hybrids fragmented mainly in the DNA part, while LNA strand fragmentation was barely noticeable. 
In summary, this study of LNA ONs demonstrated that the gas phase of DNA and LNA in both SSs and DSs differs to a great extent.

\section{References}

1. McLuckey SA, Van Berkel GJ, Glish GL. Tandem mass spectrometry of small, multiply charged oligonucleotides. J Am Soc Mass Spectrom. 1992;3:60-70.

2. Pan S, Verhoeven K, Lee JK. Investigation of the initial fragmentation of oligodeoxynucleotides in a quadrupole ion trap: charge level-related base loss. J Am Soc Mass Spectrom. 2005;16(11):1853-1865.

3. McLuckey SA, Habibi-Goudarzi S. Ion trap tandem mass spectrometry applied to small multiply charged oligonucleotides with a modified base. J Am Soc Mass Spectrom. 1994;5(8):740-747.

4. McLuckey SA, Vaidyanathan G. Charge state effects in the decompositions of singlenucleobase oligonucleotide polyanions. Int J Mass Spectrom Ion Process. 1997;162:1-16.

5. Favre A, Gonnet F, Tabet JC. Location of the negative charge(s) on the backbone of single-stranded deoxyribonucleic acid in the gas phase. Eur J Mass Spectrom (Chichester). 2000;6:389-396.

6. Wu J, McLuckey SA. Gas-phase fragmentation of oligonucleotide ions. Int J Mass Spectrom. 2004;237:197-241.

7. Schurch S, Tromp JM, Monn ST. Mass spectrometry of oligonucleotides. Nucleosides Nucleotides Nucleic Acids. 2007;26(10-12):1629-1633.

8. Cerny RL, Tomer KB, Gross ML, Grotjahn L. Fast atom bombardement combined with tandem mass spectrometry for determining strcutures of small oligonucleotides. Anal Biochem. 1987;165:175-182.

9. Sannes-Lowery KA, Hofstadler SA. Sequence confirmation of modified oligonucleotides using IRMPD in the external ion reservoir of an electrospray ionization Fourier transform ion cyclotron mass spectrometer. J Am Soc Mass Spectrom. 2003;14(8): 825-833.

10. Keller KM, Brodbelt JS. Collisionally activated dissociation and infrared multiphoton dissociation of oligonucleotides in a quadrupole ion trap. Anal Biochem. 2004;326(2): 200-210.

11. Klassen JS, Schnier PD, Williams ER. Blackbody infrared radiative dissociation of oligonucleotide anions. J Am Soc Mass Spectrom. 1998;9(11):1117-1124.

12. Ickert S, Riedel J, Beck S, Linscheid MW. Negative Nucleotide lons as Sensitive Probes for Energy Specificity in Collision Induced Fragmentation in Mass Spectrometry. Rapid communications in mass spectrometry : RCM. 2018;32(7):597-603.

13. Wang Z, Wan KX, Ramanathan R, Taylor JS, Gross ML. Structure and fragmentation mechanisms of isomeric T-rich oligodeoxynucleotides: a comparison of four tandem mass spectrometric methods. J Am Soc Mass Spectrom. 1998;9(7):683-691.

14. Yang J, Hakansson K. Fragmentation of Oligoribonucleotides from Gas-Phase IonElectron Reactions. J Am Soc Mass Spectrom. 2006;17:1369-1375.

15. McLaffety FW, Horn DM, Breuker K, et al. Electron Capture Dissociation of Gaseous Multiply Charged lons by Fourier Transfrom Ion Cyclotron Ressonance. J Am Soc Mass Spectrom. 2001;12:245-249.

16. Hari Y, Leumann CJ, Schürch S. What Hinders Electron Transfer Dissociation (ETD) of DNA Cations? J Am Soc Mass Spectrom. 2017;28:2677-2685.

17. Smith SI, Brodbelt JS. lectron transfer dissociation of oligonucleotide cations. Int J Mass Spectrom. 2009;283:85-93. 
18. Gabelica V, De PE. Comparison of the collision-induced dissociation of duplex DNA at different collision regimes: evidence for a multistep dissociation mechanism. J Am Soc Mass Spectrom. 2002;13:91-98.

19. Gabelica V, E. DP. Collision-induced dissociation of 16-mer DNA duplexes with various sequences: evidence for conservation of the double helix conformation in the gas phase. Int J Mass Spectrom. 2002;219:151-159.

20. Fabris D, Kellersberger KA, Wilhide JA. Higher-order structure of nucleic acids in the gas phase: top-down analysis of base-pairing interactions. Int J Mass Spectrom. 2012;312:155-162.

21. Lippens JL, Ranganathan SV, D'Esposito RJ, Fabris D. Modular calibrant sets for the structural analysis of nucleic acids by ion mobility spectrometry mass spectrometry. Analyst. 2016;141(13):4084-4099.

22. Porrini M, Rosu F, Rabin C, et al. Compaction of Duplex Nucleic Acids upon Native Electrospray Mass Spectrometry. ACS Cent Sci. 2017;3(5):454-461.

23. Baker ES, Bowers MT. B-DNA helix stability in a solvent-free environment. J Am Soc Mass Spectrom. 2007;18(7):1188-1195.

24. Gidden J, Ferzoco A, Baker ES, Bowers MT. Duplex formation and the onset of helicity in poly $\mathrm{d}(\mathrm{CG}) \mathrm{n}$ oligonucleotides in a solvent-free environment. $\mathrm{J}$ Am Chem Soc. 2004;126(46):15132-15140.

25. Frieden M, Christensen SM, Mikkelsen ND, et al. Expanding the design horizon of antisense oligonucleotides with alpha-L-LNA. Nucleic Acids Res. 2003;31(21):6365-6372.

26. Frieden M, Hansen HF, Koch T. Nuclease stability of LNA oligonucleotides and LNA-DNA chimeras. Nucleosides Nucleotides Nucleic Acids. 2003;22(5-8):1041-1043.

27. Wengel J, Petersen M, Frieden M, Koch T. Chemistry of locked nucleic acids (LNA): Design, synthesis, and bio-physical properties. Lett Pept Sci. 2003;10:237-253.

28. Obika S, Nanbu D, Hari Y, et al. Synthesis of 2'-0,4'-C-methyleneuridine and -cytidine. Novel bicyclic nucleosides having a fixed C-3,-endo sugar puckering. Tetrahedron Lett. 1997;38:8735-8738.

29. Koshkin AA, Singh SK, Nielsen P, et al. LNA (Locked Nucleic Acids): Synthesis of the adenine, cytosine, guanine, 5-methylcytosine, thymine and uracil bicyclonucleoside monomers, oligomerisation, and unprecedented nucleic acid recognition. Tetrahedron. 1998;54:3607-3630.

30. Petersen $M$, Wengel J. LNA: a versatile tool for therapeutics and genomics. Trends Biotechnol. 2003;21(2):74-81.

31. Huang TY, Kharlamova A, McLuckey SA. Ion trap collision-induced dissociation of locked nucleic acids. J Am Soc Mass Spectrom. 2010;21(1):144-153.

32. Allen SJ, Giles K, Gilbert T, Bush MF. Ion mobility mass spectrometry of peptide, protein, and protein complex ions using a radio-frequency confining drift cell. Analyst. 2016;141(3):884-891.

33. von Helden G, Kemper PR, Gotts NG, Bowers MT. Isomers of Small Carbon Cluster Anions: Linear Chains with up to 20 Atoms. Science. 1993;259(5099):1300-1302.

34. Revercomb HE, Mason EA. Ion mobility mass spectrometry of peptide, protein, and protein complex ions using a radio-frequency confining drift cell. Analyst. 2016;141:884-891.

35. Ickert S, Hofmann J, Riedel J, Beck S, Pagel K, Linscheid MW. Charge-induced geometrical reorganization of DNA oligonucleotides studied by tandem mass spectrometry and ion mobility. Eur J Mass Spectrom (Chichester). 2018;24(2):225-230.

36. Gabelica V, De Pauw E. Comparison of the Collision-Induced Dissociation of Duplex DNA at Different Collision Regimes: Evidence for a Multistep Dissociation Mechanism. J Am Soc Mass Spectrom. 2002;13:91-98.

37. Pan S, Sun X, Lee KL. DNA Stability in the Gas Versus Solution Phases: A Systematic Study of Thirty-One Duplexes with Varying Length, Sequence, and Charge Level. J Am Soc Mass Spectrom. 2006;17:1383-1395.

38. Wan KX, Gross ML, Shibue T. Gas-Phase Stability of Double-Stranded Oligodeoxynucleotides and Their Noncovalent Complexes With DNA-Binding Drugs As 
Revealed by Collisional Activation in an Ion Trap. J Am Soc Mass Spectrom. 2000;11:450-457.

39. Burmistrova A, Gabelica V, Duwez AS, De Pauw E. Ion mobility spectrometry reveals duplex DNA dissociation intermediates. J Am Soc Mass Spectrom. 2013;24(11): 1777-1786.

40. Gidden J, Baker ES, Ferzoco A, Bowers MT. Structural motifs of DNA complexes in the gas phase. Int J Mass Spectrom. 2005;240(3):183-193. 


\section{Figure captions}

Fig. 1 Oligonucleotide fragmentation nomenclature according to ${ }^{1}$.

Fig. 2. Structure of a LNA nucleotide (left) in comparison to a DNA nucleotide (right).

Fig. 3. a) Correlation between the charge and the fragmentation energy of the DNA or LNA SS A1. Analyses were repeated three times with differences in the relative CE needed of only $0.2 \%$. b) Correlation of the CCSs to the charge state of the DNA or LNA SS ON A1. A maximum deviation of $0.11 \%$ for the CCSs was observed. Error bars depict standards deviations of three independent analyses.

Fig. 4. ESI-MS/MS spectra of the 4-mer ON 5'-GACT-3'. Left) CID spectra of the charge states 1- (a), 2- (c) and 3- (e) of the LNA ON. Right) CID spectra of the charge states 1- (b), 2- (d) and 3- (f) of the DNA ON.

Fig. 5. Spectra of the 8-mer DNA DS ON 5'-CGG ATT AA-3'/5'-TTA ATC CG-3' in CID experiments. a) CID spectrum of the five-fold negatively charged duplex; b) CID spectrum of the four-fold negatively charged duplex; c) CID spectrum of the three-fold negatively charged duplex; d) Magnification of the range of $\mathrm{m} / \mathrm{z}$ 1400-1550 of c). The precursor ion is highlighted in purple, the signals of the intact SSs are highlighted in blue and red. Numbers indicate fragments of SSs, letters refer to fragments of the duplex.

Fig. 6. CID spectra of the five-fold negative charged nucleic acid duplexes $5^{\prime}$-GGC TAA CGC ATA GGC-3'/5'-GCC TAT GCG TTA GCC-3' (F1+F2). a) spectrum of the DNA-DNA duplex; b) magnification of a) in the range $\mathrm{m} / \mathrm{z} 1700-1830$; c) spectrum of the DNA-LNA duplex; d) magnification of c) in the range $\mathrm{m} / \mathrm{z} 1820-1930$. 
Fig. 7. Relative intensities of the dominant fragments of the five-fold negatively charged precursor ion of the 15-mer 5'-GGC TAA CGC ATA GGC-3'/5'-GCC TAT GCG TTA GCC-3' $(\mathrm{F} 1+\mathrm{F} 2)$ duplex in correlation to the relative CE applied. a) DNA-DNA duplex. b) DNA-LNA hybrid duplex. SS indicates a complete single strand signal. All charges reflected the losses of the corresponding number of protons during ionization and are not explicitly given here.Table

Table 1. Number of observed fragments above 5\% relative intensity over six charge states from the 15-mer ON 5'-GGC TAA CGC ATA GGC-3' (F1). The identical sequence was employed as LNA and DNA ONs at a relative CE of $35 \%$.

\begin{tabular}{ccc}
\hline & $\begin{array}{c}\text { Number of fragments } \\
\text { Charge state }\end{array}$ & $\begin{array}{c}\text { Number of fragments } \\
(\text { LNA })\end{array}$ \\
\hline $9-$ & 45 & 22 \\
$8-$ & 50 & 13 \\
$7-$ & 67 & 26 \\
$6-$ & 68 & 29 \\
$5-$ & 147 & 35 \\
$4-$ & 174 & 39 \\
\hline
\end{tabular}

